ENTREVISTA

\title{
Santé et Maladie: Le Point de \\ Vue Sociologique
}

Escola Nacional de Saúde PúbliCa - FIOCRUZ - RJ.
Maria Teresa de Oliveira

Une interview avec Claudine Herzlich

Si vous voulez bien commencer par nous dire quelques mots sur votre formation professionelle?

C.H. Jài commencé par une formation en psychologie. J'ai été formée en psychologie par l'Université de Paris et j'ai commencé à travailler comme assistante dans un laboratoire de recherches en psychologie sociale. Le patron du laboratoire travaillait sur un thème de recherches qui était les "répresentations collectives", une notion qui est issue de Durkheim et qui vise à étudier les mécanismes de la pensée collective en tant qu'elle diffère de la pensée individuelle et s'impose et conditionne dans une certaine mesure la pensée individuelle. Je voulais faire une thèse sur ce domaine, je cherchais un sujet précis de recherches et, un peu par hasard, je me suis orientée vers le thème de la santé et de ta maladie. En effet, j'ai assisté au cours de Claude LéviStrauss où il parlait des mythes indiens de l'origine des maladies. C'est ce qui m'a fait penser que, de la même façon que dans les sociétés traditionnelles, il y a dans les sociétés industrielles une image de la maladie qui l'inclut dans l'ensemble du système de valeurs, de l'organisation politique et du système de pensée de ces sociétés: on pouvait donc aussi travailler de la même façon sur une société industrielle moderne. J'ai donc travaillé sur ce domaine et ensuite je me suis rendu compte qu'il y avait beaucoup d'autres orientations de recherches pour les sciences sociales en rapport avec la santé et la maladie.

Mon travail a pris, petit à petit, une orientation plus sociologique. Entretemps j'avait moi-même été récrutée en tant que chercheur par le C.N.R.S., Centre National de la Recherche Scientifique, qui est la plus grande institution pour la recherche en France. J'ai continué dans ce domaine de façon à ce moment-lá autonome, en quittant ce laboratoire de psychologie sociale et en passant dans une section de sociologie. 
Disons que ce sont là les premières étapes de ma carrịère. Jusque là je suis restée très longtemps dans ce centre d'études des mouvements sociaux. Au cours de l'année qui vient de s'écouler, j'ai crée moi-même un centre de recherches sur les problèmes de médecine-maladie et sciences so. ciales qui s'appelle CERMES, Centre de Recherches de Médecine-Maladie et Sciences Sociales. Le CERMES regroupe des sociologues, des économistes et des anthropologues et est, d'autre part, soutenu financièrement par plusieurs institutions, comme le C.N.R.S., L'INSERM qui est l'institut de recherche pur la recherche médicale, et aussi par l'Ecole des Hautes Etudes en Sciences Sociales qui est le niveau post-gradué pour les sciences sociales.

Ainsi votre interét pour la snaté a commencé un peupar hasard...

Il a commencé par hasard, mais ensuite je me suis rendu compte que c'était un domaine extrêmement riche auquel on pouvait s'interesser très longtemps. Personnellement je lui vois plusieurs interêts. D'une part il est évident que c'est un problème social important, deuxièmement quelqu'un qui s'interesse aux sciences sociales peut à travers des problèmes de la santé et de la maladie aborder vraiment la plupart des grands problèmes sociaux. Le problème de la santé est une espèce de clé, de fil conducteur extrêmement intéressant pour étudier, de façon générale, les problèmes de la societé. La santé et la maladie ne m'in. téressent pas seulement pour elles mêmes, mais vraiment en tant qu'elles constituent un mode d'entrée. Pour les sciences sociales la sánté n'est pas seulement un domaine d'application, il ne s'agit pas seulement d'appliquer le débat sur des théories des sciences sociales à la santé, mais il s'agit aussi de se demander quelle vue plus générale le détour par la santé permet d'aborder. Par exemple, je pense qu'étudier le problème de la maladie et de la médecine peut finalement conduire à étudier aussi le rapport que les individus et les groupes sociaux ont avec la science: comment est-ce que la plupart des gens rencontrent concretèment le développment scientifique et l'expertise scientifique dans leur vie quoti. dienne?

Le troisième aspect est le caractère interdépendant des études sur la maladie et la santé. J'ai commencé par être psycholoque, je suis devenue sociologue et je me suis toujours interessée aux rencontres entre disciplines, de là le caractère pluridisciplinaire du centre que j'ai fondé, où je travaille avec des anthropologues, des historiens, etc. En ce sens j'ai toujours trouvé que la santé et la maladie étaient un domaine intéressant, parce que, justement, il était nécessaire de l'aborder par plusieurs disciplines. 


\section{Pourquoi le Brésil?}

Pour plusieurs raisons. L'une d'elles c'est que je connaissais Maria Andreá Loyola, avec qui j'avais eu un bon contact. Disons que on est plus attiré par queiqu'un qu'on connait et qu'on estime. D'autre part ça m'intéressait peut être particulièrement de venir au Brésil parce que, à plusieurs reprises dans les années qui se sont écoulées, j'ai eu des demandes d'étudiants brésiliens qui m'écrivaient en me demandant s'ils pouvaient venir faire une thèse en France avec moi. Les premières fois j'ai répondu non. Je leur ai écrit une let. tre en leur disant que j'étais tout à-fait désolée, que je ne connaissais pas le Brésil, et ne savais rien de la situation bré. silienne, je ne pouvais donc pas diriger une thèse sur les pro. blèmes de la santé au Brésil. J'ai répondu ça quatre ou cinq fois. Mais un jour j'ai reçu une autre lettre et là je me suis posé un problème. Je me suis dit que c'était un peu trop facile de dire non et $j$ 'ai décidé de faire une expérience, essayer une fois pour voir. J'ai alors accepté une étudiante de Belo Horizonte qui voulait travailler sur les réprésenta. tions de la santé et de la maladie dans une "favela" de Belo Horizonte. Elle est venue, j'ai eu un très bon contact avec elle, j'ai dirigé sa thèse et j'ai eu beaucoup de difficultés par. ce que, justement, je ne connaissais pas grand chose sur le Brésil, parce que l'essentiel de la littérature est portugaise et je ne lis pas le portugais. Il a donc fallu qu'elle m'appren. ne un peu quelle était la réalité brésilienne. Je me suis rendu compte que pendant très longtemps je ne comprennais pas un certain nombre de choses qu'elle voulait dire. Pen. dant ces jours derniers où je suis allée à Belo Horizonte et j'ai visité la "favela" qu'elle a analysé, j'ai mieux compris, en la voyant, certaines choses de sa thèse. Pour elle. je crois que le fait d'être venue en France avait des bons côtés et des mauvais côtés. J'ai certainement fait par rapport à elle, justement parce que je ne connaissais rien, un effort de soutien et d'encadrement encore plus grand que je ne fais d'habitude. D'un autre coté aussi, pour elle, c'était certai. nement profitable d'avoir quelqu'un qui s'étonnait de cer. taines choses qui pouvaient lui paraître évidentes et lui disait qu'est-ce que ça veut dire? pourqui? je ne comprends pas, etc.

A partir de là j'ai développé un intérêt pour le Brésil. Je me disais que ça pouvait être porfitable de développer des échanges avec d'autres étudiants qui voulaient venir travailler en France et qu'à ce moment là, il était pour moi intéressant de connaitre la realité brésilienne. D'autre part j'aime bien enseigner et aussi connaître quelque chose de nouveau.

Que pensez-vous de votre experience professionelle au Brésil? 
D'abord j'ai été très contente d'être invitée et du contact avec les gens. J'ai aussi été contente de faire ce cours. Mais à ce propos si je peux dire un certain nombre de choses qu'il ne faut pas comprendre de façon critique, je crois que les deux institutions, car je parle à la fois pour l'IMS et pour l'Ecole Nationale de Santé Publique, pourraient profiter d'avantage de cette ressource que sont les professeurs visitants. Dans mes séminaires je fais toujours un effort pour m'ajuster à mon public. Là il y avait quand mème un peu de difficultés, car je crois qu'il faut comprendre que les professeurs étrangers n'ont pas du tout de connaissances en particulier du Brésil, de son système de santé et que, peut être, quand vous les faites venir, ça vaudrait la peine de passer une demi journée ou une journée avec eux à un peu leur expliquer la realité brésilienne. $\mathrm{Par}$ exemple, une fois j'ai fait un séminaire de santé publique en Guyane Française. La Guyane c'est aussi une réalité très particulière que je ne connaissais pas. C'est effectivement l'Amérique du Sud par rapport à la France même s'il y a le système français. Il y a des indiens, des mulâtres, il y a la forêt, les difficultés de communication. Avant le séminaire qui devait durer trois jours, un séminaire très intense, les organisateurs nous ont demandé de venir deux jours avant et, pendant ces deux jours, ils nous ont fait visiter certaines choses tout en nous donnant des explications et des renseig. nements. Je pense que les enseignants ont besoin de mieux comprendre la realité sociale et le milieu culturel auquel ils doivent s'adapter.

La deuxième chose c'est évidemment le problème de la langue. Moi, par exemple, je ne parle pas portugais. Or, le problème de la traduction est en soi quelque chose de très difficile.

Je suis sûre que tout ce que rous avez apporté est très valable. Ici au Brésil nous sommes quand même assez isolés, pas seulement au point de vue physiqye mais encore au point de vue des distances internes même... Je pense que vous n'avez pas perdu votre temps, ni le nôtre.

Je n'ai pas du tout le sentiment d'avoir perdu mon temps. Je pense qu'il y a des gens qui ont été vraiment intéressés. Pour certains d'entre eux il est probable que ça leur apportera quelque chose dans leur travail. Néanmoins nous pouvons tous essayer de profiter mieux de ces recontres. D' autre part je me suis rendu compte d'une autre difficulté. C'est que les gens ici sout complétement submergés de travail. Je ne rendais bien compte, par excmple, que ceux là qui venaient au cours devaient choisir entre ceci ct celà, que tout le monde avait un cours ou une réunion. Les gens

Cadernos de Saúde Pública, R.J., 3 (3):306-314, julyett, 1987 
sont effectivement très pris. Ça m'a beaucoup frappée aussi. Il y a peu de personnes, dans le domaine de la santé envisagée dans un sens social, peu de personnes clé, très experimentées et elles sont donc complétement submergées. C'est une difficulté que je ne sais pas comment vous pouvez la resoudre.

Changeons de sujet, voulez vous? Est-ce que vous pourriez parler un peu du cours que vous êtes venue donner à Rio?

Comme je vous le disais, j'ai pris comme thèmes de cours les thèmes sur lequels $j$ 'ai travaillé. Il $y \mathrm{a}$ un premier thème qui est celui des réprésentations sociales de la santé et de la maladie. J'ai travaillé sur ce thème assez longtemps en faisant une première étude qui consistait à interviewer des individus dans un public de classe moyenne, essentielle. ment à Paris mais aussi dans un petit village de la campagne, sur la façon dont ils conçoivent les problèmes de la santé et de la maladie; sur ce qu'elles sont pour eux, comment est-ce qu'on les définit, quels sont leurs critères, comment se represente-t on les causes de la maladie, comment doit-on se conduire en regard de la santé par rapport à la maladie quand on est malade, etc. C'était une première étude qui a été publiée il y a déjà longtemps, à Paris, en 1969, et en Anglais en 1973. Le titre du livre était "Santé et maladie - analyse d'une réprésentation sociale". Ce sujet je l'ai repris par la suite, au cours d'un séminaire avec des anthro. pologues où on s'est éfforcé d'analyser quelles étaient les rencontres et articulations entre les conceptions de la maladie dans les sociétés industrielles modernes et dans les sociétés traditionelles africaines. Ce séminaire a donné lieu à un autre livre, un livre collectif, un recueil d'articles qui s'appelle "Le sens du mal" qui avait comme sous.titre "Anthropologie et histoire, sociologie de la maladie".

J'ai continué à travailler sur ce problème de la maladie avec une collègue quis s'apelle Jeanine Pierret, dans un sens plus historique, c'est-à-dire, que on s'est demandé comment avaient évolué historiquement les réprésentations de la ma. ladie, le système de significations attribué à la maladie dans différentes sociétés sur le plan historique et aussi comment avait évolué le sens donné à la notion de malade et le statut social du malade. On a essayé d'étudier ce probleme histo. riquement, non pas en faisant nous-mêmes des recherches historiques mais en utilisant des travaux des historiens, d'une part, et de l'autre en utilisant tous les documents qu'on pouvait trouver où des malades parlent de leurs maladies. Nous avons pris ce que l'on appelle des "documents personnels", les journaux intimes, le courrier, les recueils de 
lettres, où des malades parlent de leur mal. Ça a donné un livre "Maladies d'hier et maladies d'aujourd'hui".

Parmi les séances que j'ai faites, il y en a eu quatre qui ont été sur ce thème. Une autre séance qui est un peu reliée à ce thème c'est celle où j'ai parlé d'une recherche qui est en cours et qui consiste en une analyse de la presse française à propos du problème du SIDA. Au fond le probléme du SIDA paraît très limité au départ. Mais si on lit la presse ou voit qu'il s'est developpé autour de lui toute une mythologie. D'ailleurs um jornaliste a dit dans un article que, pendant un certain temps, il y a eu plus de médecins qui s'en occuppent qu'il n'y avait de malades. C'etait au début un phenomène très limité, sur lequel s'est développée cette idée d'une me nace qui vraiment atteint l'humanité toute entière, on parle même de la nouvelle peste à ce propos. J'ai alors essayé de montrer comment on peut dire que la presse, dans un cas comme ceci, construit un phénomène qui est celui du SIDA mais que, si elle construit ce phénomène, c'est effectivemént en utilisant quelque chose qui ne vient pas de la presse elle même et qui sont justement les réprésentations collectives qui s'attachent à la santé et à la maladie, en particulier à certains types de maladies. A ce propos il y a le souvenir des grandes épidémies collectives qui ont effectivement un rôle de catastrophes universelles. J'essaye d'analyser au fond pourquoi ce thème de la catastrophe collective a pu renaitre avec cette rapidité et cette force à propos d'un phénomène comme le SIDA, pas du tout pour y adhérer car, malgré la gravité du SIDA, c'est décidement quelque chose qui a des aspects très mystificateurs, mais pour en montrer les mecanismes.

Est-ce que votre analyse de la presse a quelque rapport qui soit avec l'approche des linguistes, de Roland Barthes par exemple?

C'est un travail qui débute seulement. J'ai essayé d'utiliser, par-ci par-là, certains concepts des gens qui travaillent dans le domaine d'études de la communication, mais pas spécialement Roland Barthes. J'ai essayé d'une part de distinguer différents types de mécanismes et d'essayer de comprendre comment est-ce-qu'ils jouaient un rôle par rapport à la construction du phenomène du SIDA. Par exemple, comme type de séquence on voit qu'il y a une première phase qui est un peu le discours de l'ignorance où la presse dit seulement "on ne sait pas". On ne sait pas qu'est -ce que c'est cette maladie, on ne sait pas quels en sont les mécanismes, on n'a pas d'hypothèse, etc. C'est assez intéressant parce que, d'une part, c'est un discours de l'ignorance et d'au. tre part on peut dire que, à ce moment-là, la presse elle 
même n'a pas de discours propre. Le "on" c'est le "on" des médecins et des chercheurs en quelque sorte et se rapporte à ce que les linguistes distinguent comme sujet de l'énoncé et sujet de l'énonciantion. Ensuite il y a un deuxième temps qui est le moment où on voit très bien la construction du phénomène SIDA. Il se construit sur deux plans. D'une part il commence à y avoir une connaissance scientifique, alors la presse se fait le relais d'une information specialisée mais, parallelement, il se construit ce que j'appelle in dis. cours culturel qui constitue le phénomène social du SIDA. Bien entendu, c'est une abstration dire "la" presse: il faudrait distinguer entre les divers types de média. Mais le jour. naliste commence à avoir un discours propre qui est celui du journalisme lui-même et n'est plus le relais du discours scientifique. On voit alors que le discours culturel puise dans le stock de réprésentations et, en même temps, dans les modèles scientifiques.

Dans un troisième temps il y a une espèce d'emballement où, à nouveau, le sujet de l'énoncé change. Mais la presse ne refléte plus le modèle scientifique et elle ne parle pas non plus en son nom propre, elle se fait le reflet d'une émotion collective qui est, au début. celle des malades et qui devient ensuite celle du grand public. Elle énonce les diverses formes de la peur qui s'installe dans le public. Dans une dernière séquence, certains médias essayent de reprendre la parole pour dire "il ne faut pas s"affoler", mais en disant ça le message est ambigü, il dit aussi "quelqu'un s'affole quel. que part". Le message est donc en lui méme contradictoire et donc inquétant. mème sil veut rassurer. C'est la, en brief, l'analyse que j’ai essayé de faire à propos du SIDA.

Il me semble que rous avez développé aussi les notions de statut de profession et de "medecines douces".?

Oul. j’ai fait aussi un autre cours qui correspond à deux autres recherches. Lune d'elles est un peu l'histoire de l'évolution de la profession médicale en France. En particulier l'histoire de la façon dont les médecins à la fin du XIX èmesiécle ont acquis ce que les sociologues appellent le statut de proficssion, qui donne un sens particulier à la notion de profession. Du point de vue sociologique, en effet. une profession n'est pas un métier comme les autres et ce n'est pas simplement un métier qui a un bon revenu et du prestige, mais il $y$ a une sorte d’autorite particulière à la profession qui vient du fait que les "professionnels" définissent certaines valeurs et certains secteurs de la vie sociale. Les médecins définisssent ce qu'est la santé, les juristes ce qu'est le droit etc... De ce fait les professionmels jouissent par rapport aux autres groupes sociaux et aux autres types

Cadernos de Saúde Pública, R.J., 3 (3): 306-314, jul/set, 1987 
de métier d'une autonomie particulière dans la gestion de leur propre activité et dans la gestion du groupe qu'ils cons. tituent. $\mathrm{Ce}$ sont les professionnels eux-mêmes qui décident qui peut ou non être admis aux études médicales, qui doit devenir médecin et ainsi de suite. Ils joiussent donc non seulement d'une autonomie, mais aussi d'un type de mandat particulier à la société qui est d'avoir en charge la valeur santé.

J'ai essayé de faire une analyse sur le type d'évolution qui s'était produite en France et ça me paraissait intéressant d'en parler ici dans l'Ecole de Santé Publique. En France on voit très bien que la façon dont les médecins on reussi à négocier et à obtenir ce type de statut est beaucoup liée au développement de la santé publique et à l'attitude des praticiens eux-mêmes à l'égard de la santé publique.

Le dernier thème dont j'ai parlé c'est celui des "médecines douces". Ce n'est pas un thème sur lequel j'ai travaillé moi-même, mais c'est un thème qui m'interesse et sur lequel il y a des travaux en France.

J'ai été invitée l'année dernière au Québec pour assister à un congrès sur les "médecines douces" où il y avait à la fois des médecins, des gens de sciences sociales, des adminis. trateurs de la santé, des praticiens des "médecines douces" des malades, des étudiants, des réprésentants d'organisations syndicales et d'institutions. La plupart de ces gens trouvaient que les "médecines douces" étaient très intéressantes en même temps que ça posait un certain nombre de problèmes. Personnellement j'ai un avis assez ambivalent sur ce sujet, car, tout en reconnaissant le type de demande qu'il y a à la base des "médecines douces", je pense que, au moins dans des pays comme la France, ceux de l'Europe de l'Ouest et l'Amérique do Nord, il y a un certain nombre de dangers. Ces dangers sont moins des dangers médicaux, ma is plutôt des dangers sos 'aux qui vont de pair, avec toutes les menaces sur les syst mes d'assurances maladies et des services collectifs autour de la santé. Les partisans des "médecines douces" en Amérique du Nord sont, souvent, opposés à l'Assurance maladie et aux services de santé sur le plan collectif. Ça me parait dangereux. Je pense qu'ici la situation est probablement assez différente. Déjà avant de venir j'avais pensé que c'était quelque chose d'intéressant à discuter ici et maintenant je le pense encore plus.

La notion de "médecines douces" a-t-elle des rapports avec les "techniques" de santé comme l'homéopathie et l'acupunture?

En France on met l'homéopathie à l'intérieur des "médicines douces", bien que l'homéopathie soit reconnue Cadernos de Saúde Pública, R.J., 3 (3): 306-314, jul/set, 1987 
là-bas. Ici aussi, d'ailleurs, c'est pour ça que j'ai l'impression que c'est quelquer chose de très important pour vous. En France on parle aussi bien d'homéopathie et d'acupunture qui sont les deux reconnues, mais aussi de la phytotérapie mesótérapie, iridotérapie, naturotérapie. Il y a vraiment toute une série de choses qui en France et aussi au Québec prend un seul titre, en quelque sorte un seul emblème, qui est celui des "médecines douces." 